\title{
Hydrophobic interactions at the phylloplane modulating adhesion, uptake and in planta fate of foliarly deposited particles
}

\author{
ASTRID AVELLAN ${ }^{1}$, BRUNO P MORAIS ${ }^{1}$, MATHEUS \\ MIRANDA $^{1}$, DIANA S DIAS ${ }^{1}$, GREGORY V. LOWRY ${ }^{2}$ AND \\ SONIA MORAIS RODRIGUES ${ }^{1}$ \\ ${ }^{1}$ Universidade de Aveiro \\ ${ }^{2}$ Carnegie Mellon University \\ Presenting Author: aavellan@ua.pt
}

Plants are the most important biomass pool of the critical zone. Their leaves represent a great surface for atmospheric contaminants deposition, but also for agrochemicals delivery. Nevertheless, few is known regarding the morphological, and physicochemical interplays modulating the uptake and transport of inorganics at the phylloplane. Notably, the route of entrance of inorganic particles into leaves is under debate. The common suposition is that particles could mainly penetrate plant leaves through their stomata while the cuticle, covering most of the leaf surface, act as a barrier that only ions, molecules or very small $(<2 \mathrm{~nm})$ particles could cross[1]. Our studies challenge this hypothesis.

Model gold nanoparticle (AuNP) of 3, 20 and 50nm coated with either citrate or PVP were deposited on superhydrophobic wheat leaves. While smaller NPs underwent increased leaf adhesion, they were not significantly taken up more. Further, from the deposited AuNP, up to $\sim 40 \%$ translocated to other plant compartments, and $\sim 15 \%$ were even exudated in the rhizosphere. These results underline that besides root uptake, foliar adhesion and uptake can contributes to inorganic accumulation in/on leaves, shoots, roots, and rhizosphere soil.

Moreover, surface properties tuned through NP coating strongly impacted leaf adhesion, but also their route of uptake, as assessed by enhanced dark-field microscopy with hyperspectral imaging, and $\mu$-X-Ray fluorescence mapping. While citrateAuNPs mainly entered the leaves through the stomata, "large" PVP-AuNP $(\sim 50 \mathrm{~nm})$ penetrated the leaves in areas devoid of stomata[2], through the cuticle.

Cuticular uptake is thus a crucial step to understand for the prediction of foliar adhesion and uptake of particles. We hypothesized that drivers might be (i) NPs surface properties, (ii) cuticular composition, and (iii) the leaf wettability. AuNPs surfaces were tuned for hydrophobicity and amphiphilicity, and deposited on leaves of four plant models presenting a range of leaf surface free energies[3]. Results highlight how these interplays influence NPs cuticular adhesion, foliar uptake and translocation, but also the knowledge gaps remaining for the prediction of inorganic particle behaviour at the phylloplane.

[1] Avellan et al (2021), Environmental Science \& Technology, Under review

[2] Avellan et al (2019), ACS nano 5:5291-5305.

[3] Avellan et al work in progress 